$\underline{\text { Preprint typeset in JHEP style. - HYPER VERSION }}$

SISSA $100 / 2000 / \mathrm{FM}$

hep-th/0010217

\title{
D-branes on Calabi-Yau manifolds and helices
}

\author{
A. Tomasiello \\ International School for Advanced Studies (SISSA/ISAS) \\ Via Beirut 2-4, 34014 Trieste, Italy \\ E-mail: tomasiel@sissa.it
}

\begin{abstract}
We investigate further on the correspondence between branes on a Calabi-Yau in the large volume limit and in the orbifold limit. We conjecture a new procedure which improves computationally the McKay correspondence and prove it in a non trivial example. We point out the relevance of helices and try to draw some general conclusions about Beilinson theorem and McKay correspondence.
\end{abstract}

Keywords: D-branes, Conformal field models in string theory, Differential and algebraic geometry. 


\section{Contents}

1. Introduction 1

2. Sheaves and quivers 2

2.1 McKay correspondence 3

2.2 Beilinson theorem 1

3. Multiple fibration resolutions and tautological bundles 5

4. Gram-Schmidt orthonormalization procedure and mutations 6

5. Explicit computation 8

5.1 The dictionary 8

5.2 Helices 9

6. McKay and Beilinson 10

7. Discussion 12

7.1 The mirrors of the helices 13

\section{Introduction}

The problem of describing D-branes in Calabi-Yau manifolds is clearly central in string theory, as a part of the general effort to understand its non-perturbative dynamics. In the large volume limit the condition on the cycles they can wrap is known [2] (for a review see (4). So-called A branes are 3 dimensional (throughout this paper we will refer to the dimension of the branes as if they were not extended in the uncompactified dimensions) and wrap special Lagrangian submanifolds; B branes, on which we will focus, are evendimensional and wrap holomorphic submanifolds. Of course this geometrical description is deemed to become more complicated as soon as one tries to travel in the interior of the moduli space; on the other hand, near the orbifold point it is possible to apply the known methods to analyze branes on an orbifold [9].

The generalization of these pictures to arbitrary points in the moduli space turns out to be based on the language of categories and homological algebra. Although still much is lacking to a complete understanding, it is remarkable that some non-trivial results have been beginning to emerge recently. As an example, a dictionary of branes between the large volume and the orbifold points has been set up, based on generalized McKay correspondence [6, 4] and Beilinson theorem [6, 8]. 
In this paper we will investigate further in this direction. Motivated by strikingly simple results in [6], we will state and prove in an example two conjectures: the first improves computationally the method, while the value of the second is mainly theoretical, in that it makes one discover the relevance of the concepts of helix and of mutation; these turn out to be related in an interesting way to a Gram-Schmidt orthogonalization procedure (actually this is already implicit in the work of Bondal [22]). We will note several nice mathematical features and coincidences in the picture we will develop, and try to draw a general lesson from it.

We begin in section 2 by reviewing needed physical and mathematical background. In section 3 and 1 we state our two main conjectures, and proceed to check them in explicit examples in section 5. There are moreover interesting mathematical consequences that can be explored; one of this is the comparison between McKay and Beilinson quivers, whose study we begin in section 6, for the case of projective spaces. Finally, section 0 is an attempt to justify a posteriori the success of our conjectures; we argue that the reason behind them should be more a physical than a mathematical one.

\section{Acknowledgments}

This paper would not have seen the light without the guide of Prof. Michael R. Douglas, who introduced me to these matters and supported me throughout this work. I also wish to thank IHES for its kind hospitality during the initial phases of this project.

\section{Sheaves and quivers}

As usual, we start from the linear sigma model approach [27]. Since in this paper we will only work with threefolds of codimension 1 , this model has $5+1$ chiral superfields $\left(Z^{i}, P\right)$, $i=1 \ldots 5$ with charges $\left(w_{i}, K \equiv-\sum w_{i}\right)$ with respect to the gauge group $U(1)$; the $D$-term and superpotential read

$$
D=\sum w_{i}\left|Z^{i}\right|^{2}-K|P|^{2}-\rho, \quad W=P \sum\left(Z^{i}\right)^{k_{i}+2} .
$$

In the $\rho>0$ phase, the moduli space of vacua reduces to a symplectic quotient easily recognizable as $\mathbb{P}^{w_{1}, \ldots, w_{5}}$, and the $F$-flatness condition becomes the equation which cuts a Calabi-Yau . In the other phase, all $Z^{i}$ have instead zero expectation value. In the extreme limits $\rho \rightarrow \pm \infty$, after integrating out the massive fields, both theories flow to superconformal theories: the first one confines to a sigma model on the Calabi-Yau , the second one becomes a Landau-Ginzburg theory; actually an orbifold thereof, due to an unbroken discrete relic of the $U(1)$ gauge group, which acts on $Z^{i}$ as $\omega^{w_{i}}$, where $\omega=e^{2 \pi i / K}$.

In both these theories we know how to describe even branes; in the geometric limit, they are holomorphic subvarieties with a holomorphic (and stable) bundle on them; in the orbifold limit, we can apply the quiver description [9], and associate them to representations of a quiver, with relations given by $F$-flatness.

On the other hand, the physical picture we have been reviewing is nothing but a resolution of singularities. Indeed, the symplectic quotient ( $D$-flatness plus gauge invariance) 
of the first phase is the exceptional locus of the resolution of the orbifold singularity of the second phase.

Given this simple observation, it is natural to think [6] that the correspondence between branes in the two theories is given by McKay correspondence [21, 16].

Really, one of the points of this paper will be that this idea is naturally connected with Beilinson theorem [3], which already turned out to be relevant in particular cases [8]. For this reason we are now going to briefly review both, of course with an eye on our applications.

\subsection{McKay correspondence}

What is classically called McKay correspondence is the correspondence between irreducible representations of a finite subgroup $\Gamma \subset S L_{2}(\mathbb{C})$ and irreducible components of the exceptional locus of the resolution of $\mathbb{C}^{2} / \Gamma$. For higher dimensions, the situation is not yet well understood; we will mainly refer, anyway, to the concrete framework of 16.

Let thus $\Gamma \subset S L_{n}(\mathbb{C})$. This inclusion means an action of $\Gamma$ on $\mathbb{C}^{n}$, and this gives in turn a singularity $\mathbb{C}^{n} / \Gamma$. Consider the irreducible representations $\rho_{k}$ of $\Gamma$, and the $n$-dimensional representation $Q$ given by $\Gamma \subset S L_{n}(\mathbb{C})$. We can define a quiver whose dots represent $\rho_{k}$, whose arrows are given by numbers $a_{i j}^{(1)}$ given by $Q \otimes \rho_{j}=\oplus a_{i j}^{(1)} \rho_{i}$ and with relations which, in the case in which $\Gamma$ is abelian and cyclic, can be written $X_{a, a+w_{i}}^{i} X_{a+w_{i}, a+w_{i}+w_{j}}^{j}=X_{a, a+w_{j}}^{j} X_{a+w_{j}, a+w_{i}+w_{j}}^{i}$ (which, in a common notation, can be written as $\left.\left[X^{i}, X^{j}\right]=0\right)$.

Let us now consider the space of representations of this quiver modulo its automorphisms,

$$
M \equiv \frac{\left\{X \in(Q \otimes \operatorname{End} \rho)^{\Gamma} \mid\left[X^{i}, X^{j}\right]=0\right\}}{\operatorname{GL}_{\Gamma}(\rho)}, \quad \operatorname{GL}_{\Gamma}(\rho) \equiv(\operatorname{End} \rho)^{\Gamma}
$$

with $\rho$ the regular representation (really this quotient should be defined more carefully as a GIT quotient [23]). This turns out to be a resolution of $\mathbb{C}^{n} / \Gamma$, generalizing the 2dimensional result of Kronheimer [18].

In a special case of this construction (like the resolutions obtained as Hilbert schemes of points [16]), we can go further. Define $P$ as the numerator of (2.2), and view it as a principal fibration $P \rightarrow M$; then we can define the bundle associated to the regular fibration $\mathcal{R} \equiv P \times{ }_{\mathrm{GL}_{\Gamma}(\rho)} \rho$, and the ones associated to the irreps $\rho_{i}, \mathcal{R}_{i}$; both are called tautological bundles.

Multiplication by the coordinates defines a complex

$$
\mathcal{R} \rightarrow Q \otimes \mathcal{R} \rightarrow \Lambda^{2} Q \otimes \mathcal{R} \rightarrow \ldots \Lambda^{n} Q \otimes \mathcal{R} \cong \mathcal{R}
$$

which can be decomposed as

$$
\mathcal{R}_{i} \rightarrow \oplus a_{j i}^{(1)} \mathcal{R}_{j} \rightarrow \oplus a_{j i}^{(2)} \mathcal{R}_{j} \rightarrow \oplus a_{j i}^{(3)} \mathcal{R}_{j} \rightarrow \ldots \mathcal{R}_{i}
$$

where $\Lambda^{k} Q \otimes \rho_{i}=\oplus a_{j i}^{(k)} \rho_{j}$. What is important for us is that this complex, that we call $S_{i}$, is exact outside the exceptional locus, and thus defines an element of its K-theory; and that these $S_{i}$ are dual to the $\mathcal{R}_{i}$ in a sense compatible with our definition of duality below. 
Thus, from this point of view we have what we wanted: a map which associates to each irrep of $\Gamma \rho_{i}$ a K-theory class on the exceptional divisor (and hence, by restriction, on the Calabi-Yau ).

\subsection{Beilinson theorem}

This construction [3], in most simple terms, allows us to decompose a bundle in terms of a "basis". The procedure works as follows: Start from a sheaf $F$ on the projective space $\mathbb{P}^{n}$, and pull it back to the product $\mathbb{P}^{n} \times \mathbb{P}^{n}$. On the latter space, there is a resolution of the structure sheaf of the diagonal, $\mathcal{O}_{\Delta}$, which reads

$$
0 \rightarrow \Lambda^{n}\left(\mathcal{O}_{\mathbb{P}^{n}}(-1) \otimes \mathcal{Q}^{*}\right) \rightarrow \ldots \rightarrow \mathcal{O}_{\mathbb{P}^{n}}(-1) \otimes \mathcal{Q}^{*} \rightarrow \mathcal{O}_{\mathbb{P}^{n} \times \mathbb{P}^{n}} \rightarrow \mathcal{O}_{\Delta} \rightarrow 0
$$

where $\mathcal{Q} \equiv \mathcal{T}(-1)$ is the universal quotient bundle. We can now tensor this resolution with $\pi_{1}^{*} F$ ( $\pi_{i}$ will be projections on both factors); then take an injective resolution $I^{\bullet \bullet}$ of this complex (which is, by definition, a double complex), and apply to it the direct image of the second projection $\pi_{2 *}$. Consider now the cohomology of the double complex $\pi_{2 *} I^{\bullet \bullet}$ obtained in this way; as usual, this can be computed by spectral sequences. There are two spectral sequences, depending on which filtration one chooses; one gives the result that the cohomology of this double complex is present only in total degree zero, and its sum is the sum of the grades of a filtration of the original $F$; the other one has $E_{1}$ term

$$
E_{1}^{p, q}=H^{p}\left(\mathbb{P}^{n}, F(q)\right) \otimes \Lambda^{-q} \mathcal{Q}^{*}
$$

To be more precise: the grades here have the range $0 \leq p \leq n,-n \leq q \leq 0$, and the cohomology whose sum corresponds to $F$ is in grades $p=-q$.

This procedure has a clear interpretation in the framework of derived categories [25, 11] as a Fourier-Mukai transform [26]. Indeed, the whole process can be seen as $\mathbf{R} \pi_{2 *}\left(\pi_{1}^{*} F \otimes\right.$ $\left.\mathcal{O}_{\Delta}\right)$, where $\mathbf{R}$ is the derived functor within the derived category; and it is clear that it is an identity from the derived category to itself.

What is interesting for us is that the double complex (2.6) has an interpretation as complex of quivers. Let us make this more precise; introduce the algebra $A \equiv \operatorname{Hom}\left(\oplus_{i=0}^{n} \mathcal{O}_{\mathbb{P}^{n}}(i)\right.$, $\oplus_{i=0}^{n} \mathcal{O}_{\mathbb{P}^{n}}(i)$ ). This is the path algebra of a quiver, that we will call Beilinson quiver (for $\mathbb{P}^{n}$ ), and which is the quiver with $n+1$ dots, $n+1$ arrows between each pair of consecutive dots, and relations as we described for the McKay quiver $\left[X^{i}, X^{j}\right]=0$. Now we interpret each line of (2.6) as a representation of this quiver as in [8], letting each $\Lambda^{i} \mathcal{Q}^{*}$ correspond to the $i$ th irreducible representation of the quiver; in this way the whole double complex is an element of the derived category $D^{b}(\bmod -A)$ of the abelian category $\bmod -A$ of representations of the quiver. In fact, this construction gives an equivalence of derived categories $D^{b}\left(\operatorname{Coh}\left(\mathbb{P}^{n}\right)\right)$ and $D^{b}(\bmod -A)$.

The latter can be extracted, from a complex which represents an object in the derived category, as the alternated sum of its terms. From this we can read the orbifold charges $n_{i}$, which equal $\chi(F(-i))=\chi(\mathcal{O}(i), F) \equiv(\mathcal{O}(i), F)$. 


\section{Multiple fibration resolutions and tautological bundles}

As we described above, McKay correspondence uses tautological bundles $\mathcal{R}_{i}$; in computations, it is in general not trivial to find them explicitly. A toric method to find them from first principles was described in [6]; it was applied there to the Calabi-Yau hypersurface $\mathbb{P}^{2,2,2,1,1}[8]$. The results were very easy as compared to the computations required to obtain them: to describe them, let us recall [5] that this Calabi-Yau is most easily analyzed as embedded in the resolution of the weighted projective space. This resolution has itself a simple toric description: the charge matrix reads

$$
\left[\begin{array}{rrrrrr}
0 & 0 & 0 & 1 & 1 & -2 \\
1 & 1 & 1 & 0 & 0 & 1
\end{array}\right],
$$

which means that it is a $\mathbb{P}^{3}$ fibration over $\mathbb{P}^{1}: \mathbb{P}\left(3 \mathcal{O}_{\mathbb{P}^{1}} \oplus \mathcal{O}_{\mathbb{P}^{1}}(-2)\right)$. Let us call $H$ the divisor corresponding to one of the first three vectors in the fan (in the same ordering of the charge matrix), and $L$ the divisor corresponding to the fourth (or fifth) vector. These are respectively given by an hyperplane in the fiber $\mathbb{P}^{3}$, and a hyperplane in the base $\mathbb{P}^{1}$. In these terms, the results of [6] for the $R_{i} \equiv \mathcal{R}_{i \mid \pi^{-1}(0)}$ read

$$
\begin{array}{ll}
R_{1}=\mathcal{O} & R_{2}=\mathcal{O}(L) \\
R_{3}=\mathcal{O}(H) & R_{4}=\mathcal{O}(H+L) \\
R_{5}=\mathcal{O}(2 H) & R_{6}=\mathcal{O}(2 H+L) \\
R_{1}=\mathcal{O}(3 H) & R_{8}=\mathcal{O}(3 H+L)
\end{array}
$$

(we changed notation with respect to [6]: there the $R_{i}$ are ordered differently and are the duals of (3.2). This is, however, taken into account by us by a change in the orthogonality condition). One immediately notices that the pattern followed is very easy: the coefficient of $L$ (which is relative to $\mathbb{P}^{1}$ ) goes from 0 to 1 , and the coefficient of $H$ (which is relative to $\mathbb{P}^{3}$ ) goes from 0 to 3 . One is naturally led to conjecture that this relationship between $R_{i}$ and multiple fibration structure is general. The precise statement is most easily described through an example: we will choose $\mathbb{P}^{6,2,2,1,1}[12]$, which is the companion example treated in $[5]$.

What we want to do is to resolve this space to obtain a multiple fibration of projective spaces. We do that in two steps. First we resolve the locus $z^{4}=z^{5}=0$, thus adding a new homogeneous coordinate $z^{6}$; then resolve again the locus $z^{2}=z^{3}=z^{6}=0$. The final fan and charge matrices read

$$
T=\left[\begin{array}{rrrrrrr}
1 & 0 & 0 & 0 & -6 & -3 & -1 \\
0 & 1 & 0 & 0 & -2 & -1 & 0 \\
0 & 0 & 1 & 0 & -2 & -1 & 0 \\
0 & 0 & 0 & 1 & -1 & 0 & 0
\end{array}\right], \quad Q=\left[\begin{array}{rrrrrrr}
0 & 0 & 0 & 1 & 1 & -2 & 0 \\
0 & 1 & 1 & 0 & 0 & 1 & -3 \\
1 & 0 & 0 & 0 & 0 & 0 & 1
\end{array}\right]
$$

This means that the resulting space has the following structure: A fibration in $\mathbb{P}^{1}$ over a base, $F_{0,-2}$, which is itself a fibration (with a notation that generalizes the standard one for Hirzebruch surfaces) in $\mathbb{P}^{2}$ over $\mathbb{P}^{1}$. In analogy with the previous case, let us call 
respectively $B, H$ and $L$ the divisors corresponding to the hyperplanes in the fiber $\mathbb{P}^{1}$, in the $\mathbb{P}^{2}$ and in the base $\mathbb{P}^{1}$. In analogy with what we observed in the previous case, there should be $2 \times 3 \times 2=12 R_{i}$ :

$$
\begin{array}{ll}
R_{1}=\mathcal{O} & R_{2}=\mathcal{O}\left(L_{2}\right) \\
R_{3}=\mathcal{O}\left(L_{1}\right) & R_{4}=\mathcal{O}\left(L_{1}+L_{2}\right) \\
R_{5}=\mathcal{O}\left(2 L_{1}\right) & R_{6}=\mathcal{O}\left(2 L_{1}+L_{2}\right) \\
R_{7}=\mathcal{O}(B) & R_{8}=\mathcal{O}\left(B+L_{2}\right) \\
R_{9}=\mathcal{O}\left(B+L_{1}\right) & R_{10}=\mathcal{O}\left(B+L_{1}+L_{2}\right) \\
R_{11}=\mathcal{O}\left(B+2 L_{1}\right) & R_{12}=\mathcal{O}\left(B+2 L_{1}+L_{2}\right) .
\end{array}
$$

This 12 is exactly the order of the singularity we started with (let us recall that the compact toric variety we are talking about here is the exceptional divisor $\pi^{-1}(0)$ of the resolution of this singularity). This is a first check of the conjecture: the fact that, for instance, the coefficient of $H$ in (3.4) ranges from 0 to 2 is fixed, in the framework of the conjecture, by the fact that it corresponds to a hyperplane in $\mathbb{P}^{2}$.

It is important to note that the one we wrote down is not, obviously, the only possible resolution of the initial weighted projective space. In particular, it is not the same which was alluded to in [5]; in that case there is only one step, and the exceptional locus is a ruled surface. There are in general, indeed, other possibilities of obtaining a multiple fibration by resolving; what makes the resolution we chose more special, and what we believe has to do with the nice fit $12=12$ we obtained above, is that this one does not change the canonical class - that is, it is crepant. We will come back to this when, at the end, we will try to learn a lesson from the "experimental" discoveries we are doing in this section.

We can describe, in any case, a rough motivation for the conjecture (apart from the suggestions coming from (3.2)). The $\mathcal{R}_{i}$ of the McKay correspondence are defined as tautological bundles on $M$, the whole non-compact resolution of $\mathbb{C}^{n} / \Gamma$. The idea is that it could even be that the relevant information is already contained on the exceptional locus $\pi^{-1}(0)$; if we are able to resolve this space, in turn, in order to reveal in its interior some projective spaces, then it is reasonable to think that the tautological bundles, restricted to these projective spaces, become powers of the tautological bundle on them. We will come back again on these ideas later, after having checked the conjecture in the example we chose, and having noted a few nice fits that make the picture more plausible.

\section{Gram-Schmidt orthonormalization procedure and mutations}

Before we actually do the specific computations, let us describe explicitly, for reasons that will shortly become clear, the general Gram-Schmidt procedure that we follow to find the $S_{i}$ such that $\left(R_{i}, S_{j}\right)=\delta_{i j}$. The first one, $S_{1}$, obviously equals $R_{1}$ itself. Next, let us notice that our bundles have the property $\left(R_{i}, R_{j}\right)=0 \forall i>j$. Then the others $S_{i}$ can be obtained as

$$
S_{2}=-R_{2}+\left(R_{1}, R_{2}\right) R_{1}
$$




$$
\begin{aligned}
S_{3}= & R_{3}-\left(R_{1}, R_{3}\right) R_{1}-\left(R_{2}, R_{3}\right) S_{2}= \\
& R_{3}-\left(R_{2}, R_{3}\right) R_{2}+\left[\left(R_{1}, R_{2}\right)\left(R_{2}, R_{3}\right)-\left(R_{1}, R_{3}\right)\right] R_{1},
\end{aligned}
$$

and so on. Of course near the end of the series we can use Serre to make the computations simpler.

This procedure is based only on the assumption that we made; however, it gets a particular meaning if further properties hold for the $R_{i}$. Namely, let us suppose that

$$
\operatorname{Ext}^{k}\left(R_{i}, R_{j}\right)=0 \quad \forall i>j, \forall k
$$

and that

$$
\operatorname{Ext}^{k}\left(R_{i}, R_{j}\right)=0 \quad \forall i \leq j, \forall k>0 .
$$

These conditions together make the $R_{i}$, by definition, an exceptional series 22. Using the second set of them, we can interpret $S_{2}$ as being given by an exact sequence

$$
0 \rightarrow S_{2} \rightarrow \operatorname{Hom}\left(R_{1}, R_{2}\right) \otimes R_{1} \rightarrow R_{2} \rightarrow 0
$$

that is, $S_{2}$ is the kernel of the natural evaluation. This is usually called a mutation 22,28 , 14] of $R_{2}$ within the exceptional series $\left\{R_{i}\right\}$, more specifically a left mutation, and noted as $L R_{2}$. In a similar way, we can interpret $S_{3}$ as the first term in the sequence

$$
0 \rightarrow S_{3} \rightarrow \operatorname{Hom}\left(R_{1}, L R_{3}\right) \otimes R_{1} \rightarrow \operatorname{Hom}\left(R_{2}, R_{3}\right) \otimes R_{2} \rightarrow R_{3} \rightarrow 0
$$

this sequence is obtained joining two sequences of the type (4.4), the first of which is

$$
0 \rightarrow L R_{3} \rightarrow \operatorname{Hom}\left(R_{2}, R_{3}\right) \otimes R_{2} \rightarrow R_{3} \rightarrow 0
$$

and defines $L R_{3}$. Using (4.5) and our assumptions we obtain

$$
\begin{aligned}
S_{3}= & R_{3}-\left(R_{2}, R_{3}\right) R_{2}+\left(R_{1}, L R_{3}\right) R_{1}= \\
& R_{3}-\left(R_{2}, R_{3}\right) R_{2}+\left[\left(R_{1}, R_{2}\right)\left(R_{2}, R_{3}\right)-\left(R_{1}, R_{3}\right)\right] R_{1}
\end{aligned}
$$

in agreement with result for $S_{3}$ in (4.2).

It is natural to ask oneself whether the series we constructed in our two examples are exceptional. As it turns out, even more is true: they are what is called a foundation of a helix. This means that the series $\left\{R_{i}\right\}_{i=1}^{n}$ can be extended infinitely in both senses, in such a way that any $n$ consecutive elements make up an exceptional series, and that the "periodicity" condition $R_{n+1}=R_{1} \otimes K^{*}$ holds.

This is not only a mathematical curiosity. Being a helix is a property at the heart of Beilinson theorem; it is thus plausible that this property is crucial in this context to find a quiver corresponding to a given sheaf on the Calabi-Yau. We will come back to this later, after having checked our first conjecture for the tautological bundles in our example $\mathbb{P}^{6,2,2,1,1}$, and proved our claim that the $R_{i}$ are a foundation of a helix in both examples $\mathbb{P}^{2,2,2,1,1}$ and $\mathbb{P}^{6,2,2,1,1}$. 


\section{Explicit computation}

\subsection{The dictionary}

We have now to check that the $R_{i}$ given in (3.4) really give the correct result for the bundles on the Calabi-Yau corresponding to the natural basis near the orbifold point. As a preliminary, we need some information about the divisors in this variety and their intersections. As we said, the Picard group is generated by three divisors $B, H, L$; we obtain the relations

$$
L^{2}=0, \quad H^{2}\left(H-2 L_{2}\right)=0, \quad B(B-3 H)=0, \quad B H^{2} L=1 .
$$

Moreover, the anticanonical divisor is $-K=2 B$. It follows that on the Calabi-Yau submanifold $Y$

$$
B_{Y}=3 H_{Y}, \quad H_{Y}^{3}=4, \quad\left(H^{2} L\right)_{Y}=2,
$$

the subscript ()$_{Y}$, which we will hereafter drop when no confusion is possible, meaning restriction to $Y$. This matches with the results of [5], and allows us to use results in the literature which we will need. In particular, from now on we denote by $h$ and $l$ the generators of the curves on $Y$, duals to $H$ and $L$ in the sense that

$$
\begin{array}{ll}
(H \cdot h)_{Y}=1, & (H \cdot l)_{Y}=0 \\
(L \cdot h)_{Y}=0, & (L \cdot l)_{Y}=1 .
\end{array}
$$

We can now find the $S_{i}$, and restrict them to $Y$. Since we have already described the method, let us just give the final result for the $\left(S_{i}\right)_{Y} \equiv V_{i}$ :

$$
\begin{array}{ll}
\operatorname{ch}\left(V_{1}\right)=1 & \operatorname{ch}\left(V_{2}\right)=-1+L \\
\operatorname{ch}\left(V_{3}\right)=-2+H-2 L+2 h+l+\frac{2}{3} & \operatorname{ch}\left(V_{4}\right)=2-H-l+\frac{1}{3} \\
\operatorname{ch}\left(V_{5}\right)=1-H+2 L+l-2 h+\frac{4}{3} & \operatorname{ch}\left(V_{6}\right)=-1+H-L-l-\frac{1}{3}
\end{array}
$$

(of course the first, integer, numbers, are element of $H^{0}$, while the final ones, fractionary, mean elements of $H^{6}$ ). We have not listed the others because $V_{i}+V_{6+i}=0$, a result which parallels similar ones for the other toric varieties and that matches the relation on the periods in the orbifold basis $\varpi_{i}+\varpi_{i+6}=0$.

From the latter relation and the mirror map found in [17, 24], we find the monodromy to be

$$
\left[\begin{array}{rrrrrr}
-1 & 0 & 1 & -2 & 0 & 0 \\
0 & 1 & 0 & 0 & 2 & 0 \\
-1 & 1 & -1 & -1 & 2 & 1 \\
1 & 0 & 0 & 1 & 0 & 0 \\
-1 & 0 & 0 & -1 & 1 & 1 \\
1 & 0 & 0 & 1 & 0 & -1
\end{array}\right]
$$


and as a consequence, acting repeatedly on the pure pure D6-brane state, we obtain the states

$$
\begin{aligned}
& v_{1}=(1,0,0,0,0,0) \\
& v_{2}=(-1,0,1,-2,0,0) \\
& v_{3}=(-2,1,-2,-1,2,1) \\
& v_{4}=(2,-1,0,4,0,-1) \\
& v_{5}=(1,-1,2,-1,-2,1) \\
& v_{6}=(-1,1,-1,-2,0,-1)
\end{aligned}
$$

and their negatives. The charges in (5.6) are listed as $\left(n_{6}, n_{4}^{1}, n_{4}^{2}, n_{0}, n_{2}^{1}, n_{2}^{2}\right)$; to complete the check, we have to compare these charges with the ones in the Chern polynomials. This is accomplished as usual by comparing the central charges in the two bases: $Z=n \cdot \Pi=$ $-\int e^{-t} \operatorname{ch}(V) \sqrt{\hat{A}(T) / \hat{A}(N)}$, where $t$ is the complexified Kähler form. We find

$$
r=n_{6}, \quad c_{1}=n_{4}^{1} H+n_{4}^{2} L, \quad \operatorname{ch}_{2}=n_{2}^{1} h+n_{2}^{2} l, \quad-\operatorname{ch}_{3}=n_{0}+\frac{13}{2} n_{4}^{1}+2 n_{4}^{2},
$$

using which the check can now be easily completed, comparing (5.4) and (5.6).

\subsection{Helices}

We will describe now the proof of the claims given at the end of section 3, that the $R_{i}$ make up a foundation of a helix both in the example of this paper and in the one given in [6]. We will limit ourselves to describe the main ideas, skipping details when they become too technicals.

The latter case is easier, so let us start by that one. What we have to do is to compute cohomology groups in toric geometry; there is a standard method to do that [10], but we find it easier (and perhaps more instructive) to use a mix of this and of other techniques. In the terminology of [6], we have to check that the bundles $k H+L, k H$ for $k=0, \ldots, 3$ and $k H-L$ for $k=1,2,3$ enjoy the property (which we will call acyclicity) $h^{i}=0 \forall i>0$. First of all, we will use a consequence of the general method [10]: a sheaf generated by its sections on a toric variety is acyclic. The condition for this to be true, by the general theory, turns out to include all bundles $k H+L$ and $k H$, but not $k H-L$. To treat these, we use an exact sequence

$$
\begin{aligned}
0 \rightarrow \mathcal{O}((k-1) H+L) \rightarrow & \mathcal{O}(k H-L) \rightarrow \\
& \mathcal{O}_{\mid(H-2 L)}(k H-L) \cong \mathcal{O}_{\mathbb{P}^{1}}(k) \bigotimes \mathcal{O}_{\mathbb{P}^{2}}(-1) \rightarrow 0 ;
\end{aligned}
$$

since $\mathcal{O}_{\mathbb{P}^{2}}(-1)$ has no cohomology, we reduce to the case previously treated.

We have to prove, in addition, that the inverses of these line bundles have no cohomology. We use this time two kinds of exact sequences, the first kind in which again the restriction to the divisor $H-2 L$ appears, the second one in which instead the restriction is to $L$. Using these, we can by a zig-zag procedure prove the result for all the bundles we need; a relevant feature is that one sees that there is a range of negative bundles with this property that is just enough bug to include those we are interested in. This is analogous to what happens with the series $\mathcal{O}(1), \ldots \mathcal{O}(n)$ in $\mathbb{P}^{n}$ : in that case the negative bundles 
from $\mathcal{O}(-1)$ to $\mathcal{O}(-n)$ have no cohomology, and $\mathcal{O}(-n-1)$ starts to have it. It seems as if the conjectural method we described to find the $R_{i}$ can be viewed as a means to construct helices on multiple fibrations of a certain type. We will see later why this is non trivial.

The second case, $\mathbb{P}^{6,2,2,1,1}$, is more complicated, but is conceptually similar, and we will be very sketchy. The bundles that we have to prove to be acyclic are now: a) $k H+L, k H$ for $k=0,1,2$ and $k H-L$ for $k=1,2$; b) $B+k H+k^{\prime} L$, for $k=-2, \ldots, 2$ and $k^{\prime}=-1,0,1$. The case a) can be reduced to an analysis on a reduced fan which is nothing but a 3dimensional analog of the $\mathbb{P}^{2,2,2,1,1}$ case that we have just seen. The case b) makes use again of a sequence very similar to (5.9), with the restriction to the divisor $B$ appearing instead of that to $H-2 L$. In that case, the divisor turned out to be isomorphic to $\mathbb{P}^{1} \times \mathbb{P}^{2}$, and we had at our disposal known vanishing theorems for line bundles on $\mathbb{P}^{n}$; in this case, the divisor $B$ is isomorphic to $\mathbb{P}^{2,2,1,1}$, and again we can use a 3 -dimensional analog of the discussion above to get the vanishing theorems needed.

Finally, we have to show that the bundles inverse to those of the cases a) and b) have no cohomology. Similar techniques to the above let us get the desired results for these bundles as well.

In both cases, what we have really shown is that the $R_{i}$ are an exceptional collection. To show that they are a foundation of a helix requires to check that $R^{(n-1)} R_{1}=R_{1}(-K)$, where $n$ is the length of the collection. About the proof of this we have nothing special to say, but that it is made easier by reformulating in terms of both left and right mutations; and that it is this last fact is very plausible from the very beginning, due to the peculiarly easy form of the series. In the $\mathbb{P}^{2,2,2,1,1}$ case, indeed, let us observe that, if one were to guess the term following $R_{8}$ in the series $(3.2)$, one would naturally write $\mathcal{O}(4 H)$, which is indeed $K^{*}$; the same is true for the case of $\mathbb{P}^{6,2,2,1,1}$, for which the natural guess after the series (3.4) would be $\mathcal{O}(2 B)$, which is again $K^{*}$.

\section{McKay and Beilinson}

Let us finally analyze the relationship between the picture that emerged in this paper and the McKay correspondence. The McKay correspondence, as we saw, gives the $S_{i}$ as complexes in terms of the tautological bundles $\mathcal{R}_{j}$ on the resolution of the singularity. The method emerged in this paper gives them, instead, intrinsically in terms of the exceptional locus, exploiting the existence of a helix on this locus to use the technique of mutations. The fact that both methods work is in itself a proof of the fact that they are compatible; however, a systematic comparison could be interesting to do.

To give an idea of what this comparison looks like, we treat the easy case of projective spaces $\mathbb{P}^{n}$. In this case, the McKay quiver has $n+1$ dots arranged cyclically with $n$ links between each pair of consecutive dots (to form a closed loop), and with the usual relations; Beilinson one, as we said, is the same but with links between dot $\{n+1\}$ and $\{1\}$ missing. Moreover, $R_{i}=\mathcal{R}_{i \mid \mathbb{P}^{n}}=\mathcal{O}_{\mathbb{P}^{n}}(i-1)$. Throughout this example, we will keep a notation

halfway from explicit and abstract, exploiting only the form of the quiver, to emphasize how to do the comparison in general. In the McKay framework,

$$
S_{1}=\mathcal{R}_{1}-a_{21}^{(1)} \mathcal{R}_{2}+a_{31}^{(2)} \mathcal{R}_{3}+\ldots+(-)^{n} \mathcal{R}_{1}
$$


as a K-theory class. We know [16] that this class has support only on $\mathbb{P}^{n}$. From the definition of helices, on the other hand, we know that the $n$th right mutation of a helix gives the helix itself. This gives in general the complex

$$
\begin{aligned}
0 \rightarrow R_{i} & \rightarrow \operatorname{Hom}\left(R_{i}, R_{i+1}\right)^{*} \otimes R_{i+1} \rightarrow \operatorname{Hom}\left(R^{(2)} R_{i}, R_{i+2}\right)^{*} \otimes R_{i+2} \rightarrow \\
\ldots & \rightarrow \operatorname{Hom}\left(R^{(n-1)} R_{i}, R_{i+n}\right)^{*} \otimes R_{i+n} \rightarrow R_{i}(-K) \rightarrow 0
\end{aligned}
$$

(which in this case is simply $\mathcal{O}-V \mathcal{O}(1)+\Lambda^{2} V \mathcal{O}(2)+\ldots+(-)^{n+1} \mathcal{O}(n+1)=0$, where $\left.V=H^{0}\left(\mathbb{P}^{n}, \mathcal{O}(1)\right)\right)$. Remember now that the noncompact manifold $M$, which is the resolution of the singularity, is the total space of the line bundle $K$ on the exceptional locus. Then we can pull back (6.2) to $M$, to find an analogous relation on the $\mathcal{R}_{i}$. Exploiting the fact that $a_{21}^{(1)}=V^{*}=\operatorname{Hom}\left(R_{1}, R_{2}\right)^{*}, a_{i+1,1}^{(i)}=\Lambda^{i} V^{*}=\operatorname{Hom}\left(R^{(i-1)} R_{1}, R_{i+1}\right)^{*}$ we can use the pull-back of (6.2) for $i=1$ on $M$ to reexpress first $n$ terms (that is, all outside the last) of (6.1) as $S_{1}=\mathcal{R}_{1}-\mathcal{R}_{1}(-K)$.

The pull-back $p^{*} K$ of $K$ to its total space $M$ has a tautological section which has a zero exactly on $\mathbb{P}^{n} \subset M$. This gives a sequence

$$
0 \rightarrow p^{*} K^{*} \rightarrow \mathcal{O}_{M} \rightarrow \mathcal{O}_{\mathbb{P}^{n}} \rightarrow 0
$$

by twisting it with $\mathcal{R}_{1}$, we obtain

$$
S_{1}=\mathcal{R}_{1}-\mathcal{R}_{1}(-K)=\mathcal{R}_{1 \mid \mathbb{P}^{n}}=R_{1}
$$

that is, we obtain that the result is supported on $\mathbb{P}^{n}$, as it should, and the same result as with the mutation method (although for this first step it is a little trivial).

A less trivial check is obtained with the second term; one uses again the pull-back of $\left(\right.$ 6.2) , this time with $i=2$. Exploiting $a_{12}^{(1)}=\Lambda^{n-1} V^{*}=V=\operatorname{Hom}\left(R_{1}, R_{2}\right), a_{i+2,2}^{(i)}=$ $\Lambda^{i} V^{*}=\operatorname{Hom}\left(R^{(i-1)} R_{2}, R_{i+2}\right)^{*}$, we can reexpress this time first $n-1$ terms of

$$
S_{2}=\mathcal{R}_{2}-a_{32}^{(1)} \mathcal{R}_{3}+a_{42}^{(2)} \mathcal{R}_{4}+\ldots+(-)^{n} \mathcal{R}_{2}
$$

as

$$
S_{2}=\mathcal{R}_{2}-V \mathcal{R}_{1}-\left(\mathcal{R}_{2}(-K)-V \mathcal{R}_{1}(-K)\right)=R_{2}-\left(R_{1}, R_{2}\right) R_{1}
$$

as it should be. It is straightforward to continue to the end this check. Let us note, by the way, that a similar method can give compact expressions for the $V_{i}$ on the Calabi-Yau in terms of the $R_{i}$.

Thus, we have shown in this example that Beilinson and McKay methods agree and give the same $S_{i}$; in doing that, we have exploited a series or relations between $a^{(i)}$ and $\operatorname{Hom}\left(R_{j}, R_{k}\right)$ that we can resume by saying that Beilinson quiver is invariant under mutations, and that McKay quiver is an extension of it, in the sense that Beilinson one is obtained by cutting arrows between two consecutive dots: this is the mathematical counterpart of what was done in [6]. In this case we already knew this fact (it was stated at the beginning of this section), but it is true in general; this is essential, as it allows to interpret the quiver representation given by Bondal construction we outlined in section 0 as a representation of the McKay quiver, and hence as a D-brane. Moreover, this fact 
seems to support and generalize the claim [8] that all holomorphic objects near the orbifold points are given by Beilinson representations, i.e. by representations of the McKay quiver whose links from the last dot and to the first one have been cut.

As a further clarification of this phenomenon, let us describe an example, again for the $\mathbb{P}^{n}$ case. The representation of the McKay quiver with all $n_{i}=1$ is the D0 on the resolution $M$. On the other hand, one can easily see from its very definition as an universal quotient, that, chosen a point $p \in \mathbb{P}^{n}$, the bundle $\mathcal{Q}$ has a section which vanishes exactly in $p$. From this we obtain a resolution

$$
0 \rightarrow \Lambda^{n} \mathcal{Q}^{*} \rightarrow \ldots \rightarrow \mathcal{Q}^{*} \rightarrow \mathcal{O}_{\mathbb{P}^{n}} \rightarrow \mathcal{O}_{p} \rightarrow 0
$$

which gives $\mathcal{O}_{p}=\oplus S_{i}=\oplus n_{i} S_{i}$, with $n_{i}=1$. The representation of the Beilinson quiver corresponding to the McKay one describes the same state but bound not to move from the exceptional locus. In this way one can, by the way, prove the relations mentioned shortly after (5.4).

\section{Discussion}

Let us now try to put all the pieces together. What we initially tried was a guess for the K-theory classes corresponding, in the large volume limit, to the orbifold basis of D-branes. In doing that, we noticed that the $R_{i}$ are not only so easy to guess in general without doing the complicated computations starting from first principles as in [6], but that they are the foundation of a helix. This cannot be a coincidence, and we want now to justify this fact a posteriori, or at least to see it from a larger perspective.

First of all, let us expand the comment about helices and Beilinson theorem that we made at the end of section 1 . We saw in section 2.2 that, on projective spaces, Beilinson procedure takes an element of the derived category and expresses it as the cohomology of a double complex, which we may then use to find a quiver, and thus the "translation" of the initial sheaf in orbifold terms. There were, in that proof, two relevant series of bundles: the $\mathcal{O}(i)$ and the $\Lambda^{j} \mathcal{Q}^{*}$. Note that these are precisely the $R_{i}$ and $(-)^{j} S_{j}$ in the case of the projective space. Of course, as far as general definitions of these two series of objects are concerned, we only know that the first ones are restrictions to $\pi^{-1}(0)$ of the tautological bundles, and the second ones are dual to them; we do not know a priori of any reason for which they could give a resolution. But, this reason is just what we found, almost by chance (see discussion below), to be true in our cases: it is the extra piece of information that $R_{i}$ are foundation of a helix (a first, though partial, proof of this fact is a theorem 113], which still refers to $\mathbb{P}^{n}$, but whose proof already seems independent enough from this assumption; a more general, though more abstract, construction, is then given in the paper by Bondal in [22]). More explicitly, the definition of Beilinson quiver we gave for $\mathbb{P}^{n}$ can be generalized as the quiver whose path algebra is $A \equiv \operatorname{Hom}\left(\oplus_{i} R_{i}, \oplus_{i} R_{i}\right)$. Then, if the $R_{i}$ are a foundation of a helix, there is an equivalence of derived categories $D^{b}\left(\operatorname{Coh}\left(\pi^{-1}(0)\right)\right) \cong D^{b}(\bmod -A)$, as for the case in which $\pi^{-1}(0)=\mathbb{P}^{n}$ that we saw above. This is the generalization of Beilinson theorem we needed, and it needs the helix property that we found! 
Let us now come back to our multiple fibration resolution. We already noted that the match between the number of $R_{i}$ found by our method and the order of $\Gamma$ is already a non trivial check. We add now that another non trivial fact is that we have found a helix on the multiple fibration. The simplest examples of multiple fibrations are given by Hirzebruch surfaces $F_{k}$; in this case [22], it is known that, in the cases $F_{k}, k>3$ there are no helices made up of line bundles. So our examples could seem to be special in two senses: 1) they yield the match we talked about above; 2) they allow helices on them made up of line bundles. One special property that our resolutions share is that they are crepant resolutions of the original $\pi^{-1}(0)$. This is linked with another, very natural, property that toric varieties can have: that of allowing a non-singular Calabi-Yau inside them. In general, indeed, it is true that one can take in any toric variety a subvariety supported on the anticanonical divisor, and that a so chosen subvariety has formally a trivial canonical bundle; but, for most ambient toric varieties, there would be no way to find a non singular Calabi-Yau in this way. The condition to find non singular CalabiYau is that the polyhedron of the toric variety, with respect to its anticanonical sheaf, be integral; and this condition in turn means that the ambient toric variety has only Gorenstein singularities, which admit a partial crepant resolution [1].

So we have found that an event which seems a priori to be very unlikely, the existence on a multiple toric fibration of a helix with the right properties, seems to take place precisely when the toric fibration admits a non singular Calabi-Yau inside. This fact is somewhat surprising, from a mathematical point of view; the reason is probably that the dictionary between sheaves on the large volume Calabi-Yau and quivers is required to exist by physics. Although we concentrated in this paper on a class of examples (in general, as stated above, the crepant resolution is only partial; and the final space after the resolution could be different from a multiple fibration), the structure found here makes it probable that helices play a role in more general cases as well [19, 12].

A final remark is that we could have even guessed that our dictionary makes use of helices, because of their mirror symmetry interpretation [14, 15]. Let us check whether we obtain a consistent result even from this point of view.

\subsection{The mirrors of the helices}

The mirror theory to the linear sigma model we are dealing with was found in [15] by a dualization procedure inspired by T-duality from the world-sheet perspective. Let us put aside, in this section, the superpotential. Before dualizing, let us modify the initial linear sigma model to take in account the resolutions of the exceptional locus. The initial model has a $U(1)$ gauge invariance and 5 chiral multiplets $Z^{i}$ (apart from $P$ ); this describes, as the exceptional locus, the toric variety $\mathbb{P}^{w_{1}, \ldots, w_{5}}$. Since we resolved the latter in a more general toric variety, we modify the charge matrix of the model in exactly the same way in which we modified the charge matrix of the toric manifold, adding chiral multiplets and gauge invariances. For instance, in the $\mathbb{P}^{6,2,2,1,1}$ case, we add two chiral and two gauge multiplets. 
The result we need is that the dual theory has a twisted superpotential of the form

$$
\widetilde{W}=\sum_{a} \Sigma_{a}\left(\sum_{i} Q_{a i} Y^{i}-\rho_{i}\right)+\mu \sum_{i} e^{-Y^{i}}
$$

where $\Sigma_{a}$ are the gauge multiplets, $Y^{i}$ are the dual twisted chiral multiplets, and $\rho_{i}$ are the FI terms. In the $\rho_{i} \rightarrow \infty$ limit, which in the original theory is the sigma model limit, the gauge multiplets become infinitely heavy and become Lagrange multiplier: we end up with a twisted superpotential $\widetilde{W}=\mu \sum_{i} e^{-Y^{i}}$ and constraints $\sum_{i} Q_{a i} Y^{i}=\rho_{i}$. In this dual theory, branes are described [14] by their images in the complex plane under the map defined by the superpotential: each brane gets mapped to a straight half-line coming out from a critical point. A physical analysis of branes in this theory allows then to see the counterparts of the helix condition - although a strange feature seems to be that one has to break supersymmetry at some stage.

The simple check we want to do here is that there is the right number of critical points - and thus, the right number of mirror branes. We will do that again for the $\mathbb{P}^{6,2,2,1,1}$ example, trying to describe the features that give the expected agreement. Defining $\lambda_{i}=e^{-\rho^{i}}$, and Lagrange multipliers $\alpha_{i}$, we obtain from the charge matrix (3.3) a system of equations:

$$
\lambda_{3}=\alpha_{3}\left(\alpha_{3}-3 \alpha_{2}\right), \quad \lambda_{2}=\frac{\alpha_{2}^{2}\left(\alpha_{2}-2 \alpha_{1}\right)}{\left(\alpha_{3}-3 \alpha_{2}\right)^{3}}, \quad \lambda_{1}=\frac{\alpha_{1}^{2}}{\left(\alpha_{2}-2 \alpha_{1}\right)^{2}} .
$$

This system has degree $2 \times 3 \times 2=12$; the critical values of the superpotential equal $\widetilde{W}=$ $2 \alpha_{3}$, which has thus 12 solutions, as it should. Explicitly we have $\alpha_{3}=\sqrt{\lambda_{3}\left(1+3 \sqrt[3]{\lambda_{2}\left(1+2 \sqrt{\lambda_{1}}\right)}\right)}$, where each of the roots is understood with multiple choice. So the 12 critical points are organized generically in 6 circles in the complex $\widetilde{W}$ plane.

This agreement is due to the following fact. The degrees of the three equations are dictated by the three rows of the charge matrix in $(3.3)$. If the negative number in the second row, for instance, were different from -3 , the exponent of the denominator in the second equation in (7.2) would be higher than 3 , and the resulting would be higher, giving more critical points than needed. The $2 \times 3 \times 2$ which came in our conjecture because of the dimensions of the $\mathbb{P}^{k}$ in the multiple fibration structure, here is transformed in the same product, but with each factor coming from the degree of an equation. These are equal because all rows of the charge matrix but the one corresponding to the fiber have zero sum of degrees, as one can check in examples. This seems to be connected again with the integrality of the polyhedron we referred to in the above discussion.

Note added. When this paper was ready for publication, a paper appeared [12] which overlaps with this work.

\section{References}

[1] V. V. Batyrev, Dual polyhedra and mirror symmetry for Calabi-Yau hypersurfaces in toric varieties, J. Alg. Geom. 3 (1994) 493-535. 
[2] K. Becker, M. Becker and A. Strominger, Five-branes, membranes and nonperturbative string theory, Nucl. Phys. B 456 (1995) 130 hep-th/9507158.

[3] A. A. Beilinson, Coherent sheaves on $\mathbb{P}^{n}$ and problems of linear algebra, Funct. Anal. Appl. 12, 214-216 (1978).

[4] I. Brunner, M.R. Douglas, A. Lawrence and C. Römelsberger, D-branes on the quintic, J. High Energy Phys. 08 (2000) 015 hep-th/9906200.

[5] P. Candelas, X.D. L. Ossa, A. Font, S. Katz and D.R. Morrison, Mirror symmetry for two parameter models. $i$, Nucl. Phys. B 416 (1994) 481 hep-th/9308083.

[6] D.-E. Diaconescu and M.R. Douglas, D-branes on stringy Calabi-Yau manifolds, hep-th/0006224.

[7] D.-E. Diaconescu and J. Gomis, Fractional branes and boundary states in orbifold theories, 円. High Energy Phys. 10 (2000) 001 hep-th/9906242.

[8] M.R. Douglas, B. Fiol and C. Römelsberger, The spectrum of BPS branes on a noncompact Calabi-Yau, hep-th/0003263.

[9] M.R. Douglas and G. Moore, D-branes, quivers and ale instantons, hep-th/9603167.

[10] W. Fulton, Introduction to Toric Varieties, Annals of Mathematical Studies 131, Princeton University Press, 1993.

[11] S. I. Gelfand and Y. I. Manin, Methods of homological algebra, Springer 1996.

[12] S. Govindarajan and T. Jayaraman, D-branes, exceptional sheaves and quivers on Calabi-Yau manifolds: from Mukai to McKay, hep-th/0010196.

[13] A. L. Gorodentsev and A. N. Rudakov, Exceptional vector bundles on projective spaces, Duke Math. J. 54, n.1, 115(1987).

[14] K. Hori, A. Iqbal and C. Vafa, D-branes and mirror symmetry, hep-th/0005247.

[15] K. Hori and C. Vafa, Mirror symmetry, hep-th/0002222.

[16] Y. Ito and H. Nakajima, McKay correspondence and Hilbert schemes in dimension three, math.AG/9803120.

[17] P. Kaste, W. Lerche, C.A. Lutken and J. Walcher, D-branes on k3-fibrations, Nucl. Phys. B

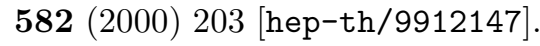

[18] P. B. Kronheimer, The construction of ALE spaces as hyper-Kähler quotients, J. Diff. Geom. 29, 665 (1989).

[19] P. Mayr, Phases of supersymmetric D-branes on Kähler manifolds and the McKay correspondence, J. High Energy Phys. 01 (2001) 018 hep-th/0010223.

[20] C. Okonek, M. Schneider, H. Spindler, Vector bundles on Complex Projective Spaces, Birkhäuser, Boston, 1980.

[21] M. Reid, La correspondence de McKay, math.AG/9911165.

[22] A. N. Rudakov et al., Helices and Vector Bundles: Seminaire Rudakov, London Mathematical Society, Lecture Note Series 148, Cambridge University Press.

[23] A.V. Sardo-Infirri, Resolutions of orbifold singularities and flows on the McKay quiver, alg-geom/9610005. 
[24] E. Scheidegger, D-branes on some one-and two-parameter Calabi-Yau hypersurfaces, J. High Energy Phys. 04 (2000) 003 hep-th/9912188.

[25] R. P. Thomas, Derived categories for the working mathematician, math.AG/0001043.

[26] R. P. Thomas, Mirror symmetry and action of braid groups on derived categories, math.AG/0001044.

[27] E. Witten, Phases of $N=2$ theories in two dimensions, Nucl. Phys. B 403 (1993) 159 hep-th/9301042.

[28] E. Zaslow, Solitons and helices: the search for a math physics bridge, Comm. Math. Phys. 175 (1996) 337 hep-th/9408133. 